\title{
The great primate debate
}

\author{
When can invasive experiments on monkeys or \\ apes be justified? And what would be the \\ consequences for biomedical research if they \\ were to cease? Sally Goodman and Erika Check \\ pose some difficult questions.
}

$T$ here can be few more emotive issues in science than experimentation on our fellow primates. To the researchers involved, such research is vital for continued scientific progress against killers such as HIV and Alzheimer's disease. To militant animal-rights activists, those who work on primates are the worst kind of animal 'abuser', deserving public vilification.

Public attitudes and regulations vary from country to country. In Britain, for instance, research on primates is highly unpopular, and tightly controlled; whereas in the United States, both public opinion and the regulatory climate are more permissive. This international variation provides an opportunity to ask some key questions about the current and future practice of scientific research on primates. Could some of the experiments that are being conducted be abandoned without significant loss? Has the unfavourable climate in countries such as Britain already impeded scientific progress?
And against this background, how might the debate over research on primates develop in the future?

Given concerns for the animals' welfare, and the fact that many primates are threatened or endangered, researchers everywhere agree that invasive experiments on primates are only justified when there is no alternative. "We should only use primates where we can be sure that our research results will be used by clinicians," adds Bert 't Hart, an immunologist at the Biomedical Primate Research Centre at Rijswijk in the Netherlands.

The difficulty of procuring primates (see 'Supply and demand', below) and the high cost of keeping them also help to ensure that such experiments are not conducted lightly. But the fact that the United States, which used some 57,000 primates in research in 2000 , uses more than five times as many of the animals as the European Union suggests that the interpretation of 'no alternative' differs from nation to nation.

\section{Supply and demand}

Throughout the world, there is a shortage of primates for research and nowhere is this more acute than in Europe. More than half of the primates used for research in the European Union come from farms in China, Mauritius, Israel and the Philippines - a situation that satisfies no one. Chinese farms, for instance, are depleting wild populations because they mainly supply first-generation offspring from wild-captured animals. And, after a campaign led by animal-rights groups, no airline will fly primates directly into Britain, Europe's biggest user. This has had little effect on the number of primates imported, but it means that some monkeys now endure a combined air and land transit of more than $\mathbf{5 0}$ hours.

Researchers working on primates argue that the solution is to establish sustainable breeding colonies in Europe. The Dutch government in April agreed to provide 40 million euros (US\$37.5 million) to the Biomedical Primate Research Centre in Rijswijk to improve its research facilities and animal housing, to look into alternatives to primates in research, and to expand its breeding programme. But that will not solve the supply problems facing researchers elsewhere.

In Japan, where the Japanese macaque (Macaca fuscata fuscata) is widely used in neuroscience research, some labs rely in part on wild monkeys caught by local authorities that regard them as agricultural pests. But pressure from animal-rights activists to stop the supply to researchers means that the monkeys are now often killed instead.

The situation in the United States
— where there is federal support for macaque breeding programmes, and there are several private-sector suppliers - is somewhat better. But demand still outstrips supply, particularly for the pathogen-free rhesus macaques needed for AIDS research, and researchers complain that government support for primate breeding fluctuates from year to year. Demand is also expected to rise, as funding pours into research to combat bioterrorism, some of which will involve primate models.

Perhaps the biggest worry is that many primates may disappear from the wild as a result of habitat destruction and hunting. Greatly expanded captive breeding might be the animals' best long-term hope of survival. That could, of course, also provide a stable supply of primates for research.

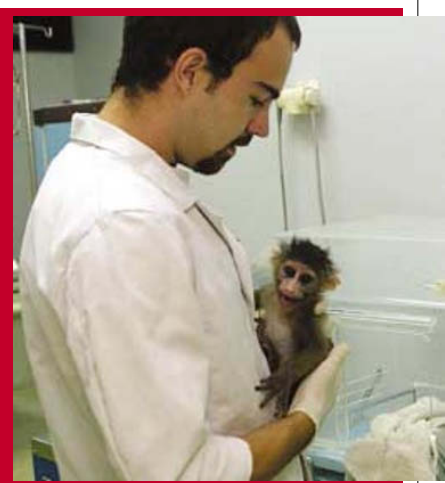

But don't bet on the scientists involved and their opponents putting aside their differences to collaborate on conservation through captive breeding. "The danger with supporting breeding centres for research is that, if animals are available, then scientists will find uses for them," says Maggy Jennings, head of the research animals department at Britain's Royal Society for the Prevention of Cruelty to Animals. 


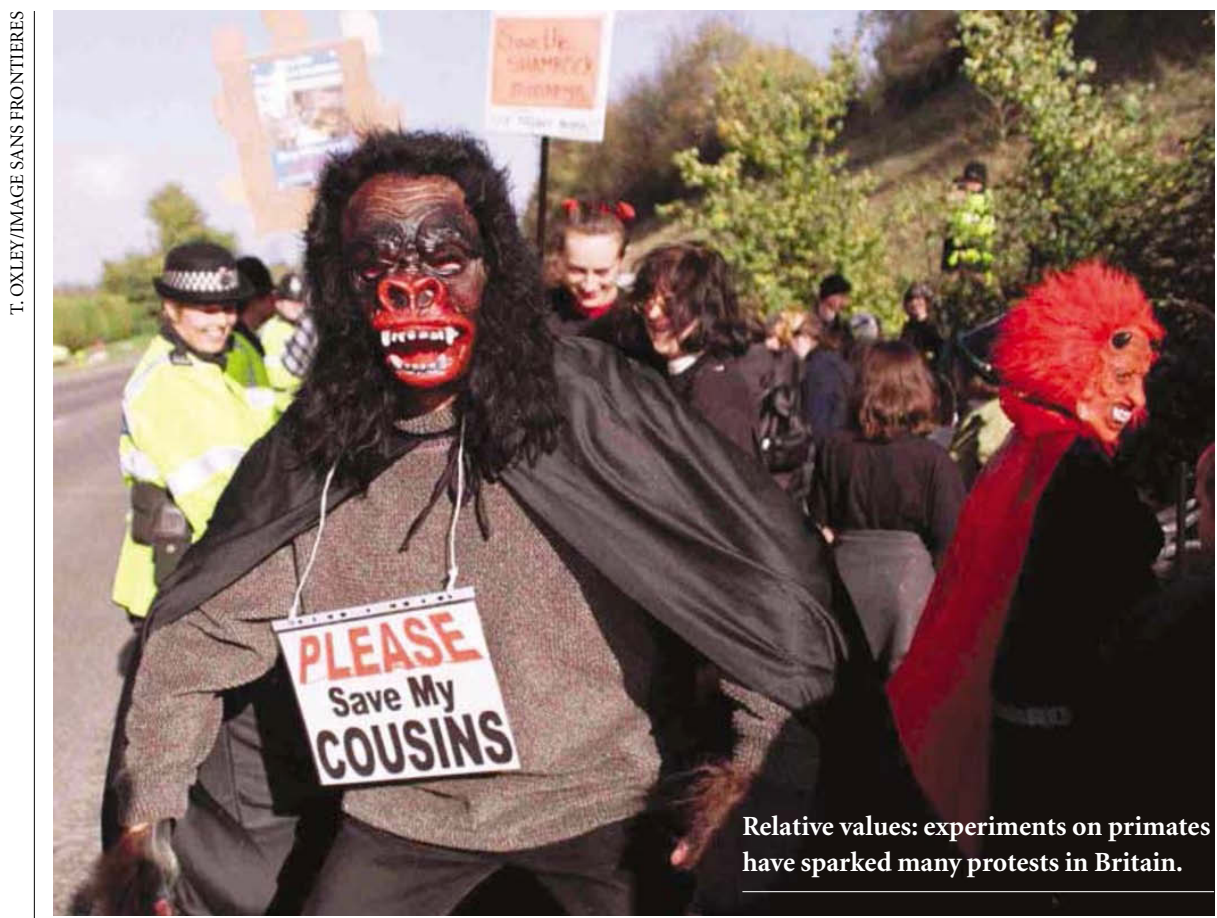

Britain, generally seen as having the world's toughest regulatory framework, is a good place to start examining the issues. And the British situation should be brought into clearer focus this week by a new series of papers from the Boyd Group, a discussion forum that aims to find common ground between biomedical scientists and antivivisectionists. It was formed in 1992 by neuroscientist Colin Blakemore of the University of Oxford and Les Ward, director of Advocates for Animals, a pressure group based in Edinburgh.

Published by the British Psychological Society, the papers cover such questions as the 'moral status' of monkeys and apes essentially, whether their capacity for social and mental suffering should single them out for special protection, or even certain 'human' rights. They also include a discussion of the use of primates in toxicity testing for drugs, which accounted for threequarters of the primates used in licensed experiments in British labs in 2000.

Most regulatory bodies require drugs to be tested on one rodent and one non-rodent species before entering human clinical trials. Usually, dogs are used, but toxicologists may also turn to primates. In the case of proteinand peptide-based drugs and vaccines which are likely to grow in importance in the postgenomic era - there may be no alternative. In such cases, a difference of a single amino acid can mean the difference between saving a cancer patient and causing liver damage, so toxicologists want a model that is as close to people as possible.

For other types of drug, there is growing evidence that models such as mini-pigs dwarf varieties that can be kept conveniently in the lab — might be just as good as monkeys.
But according to the Boyd Group, some companies are still using primates when dogs do not fit the bill because they fear that regulators, who have little experience with alternative models, might send back the application with a request for monkey data.

\section{Better by design}

The Boyd Group advocates more discussion and negotiation with regulatory authorities early in the design of toxicity studies of new drugs to ensure that primate use is really justified. Other experts argue that there is potential for reducing the number of primates used by giving more consideration to experimental design and statistical methods. But the Boyd Group's papers caution that a sudden move to ban primate use in Europe might simply lead to the work being relocated to countries where regulations are less restrictive, such as China.

Toxicology is not the only area where some researchers feel that there is scope to reduce primate use. Pierre Druilhe, a malaria researcher at the Pasteur Institute in Paris, believes researchers in his field sometimes opt for primate models for fallacious
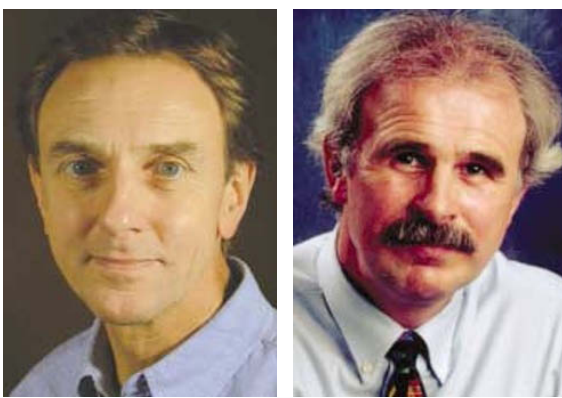

Colin Blakemore (left) and Les Ward are seeking common ground for science and animal rights. anthropomorphic reasons. The relationship between host and parasite is the product of countless generations of co-evolution, Druilhe notes. So for certain aspects of the work, it may be better to study a naturally occurring host-parasite interaction in a rodent than, for example, infecting monkeys with the human malaria parasite.

But similar evolutionary arguments also mean that primate models are the only viable option for research on some infectious diseases. Research into HIV vaccines is a case in point. Here, pathogen-free rhesus macaques (Macaca mulatta) are regarded as an essential model. When infected with simian immunodeficiency virus (SIV), a close relative of HIV, they become ill very rapidly. The symptoms closely mirror human AIDS.

"Monkey models are particularly valuable when you want to introduce genetically defined viruses into the animal and ask what are the consequences," says Joe Sodroski, director of the Center for AIDS Research at the Dana-Farber Cancer Institute in Boston. And in recent years, researchers have developed genetically engineered versions of SIV dubbed SHIV, containing genes for proteins from HIV that are recognized by the immune system. This has made the rhesus macaque even more suitable for vaccine studies.

One important insight from monkey research has been that some vaccine strategies that are applied to other diseases - those that stimulate primarily an antibody response seem unlikely to work against HIV. One such vaccine has entered clinical trials, and few AIDS researchers rate its chances of success. But without macaque models, researchers might still be making intense efforts in this direction, potentially wasting large sums of money and exposing human volunteers to the risks of side effects while failing to protect them from infection. Significantly, several vaccine strategies that were developed in macaques are now moving into human

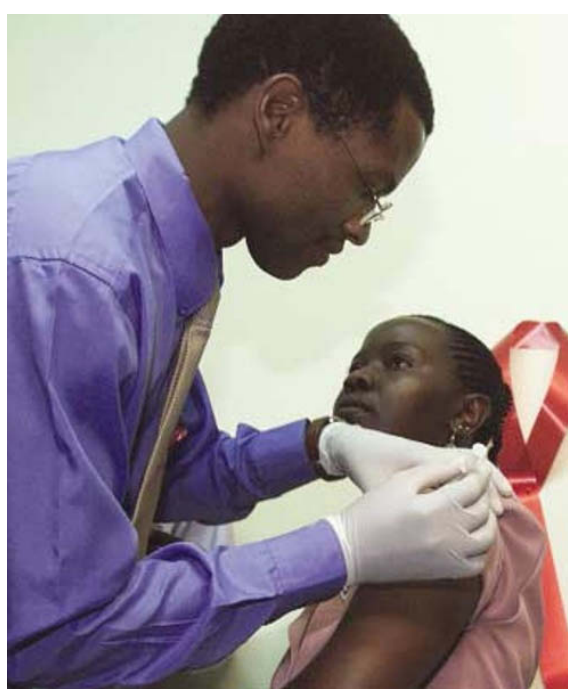

Without primate research, would candidate vaccines for HIV now be ready for trials? 


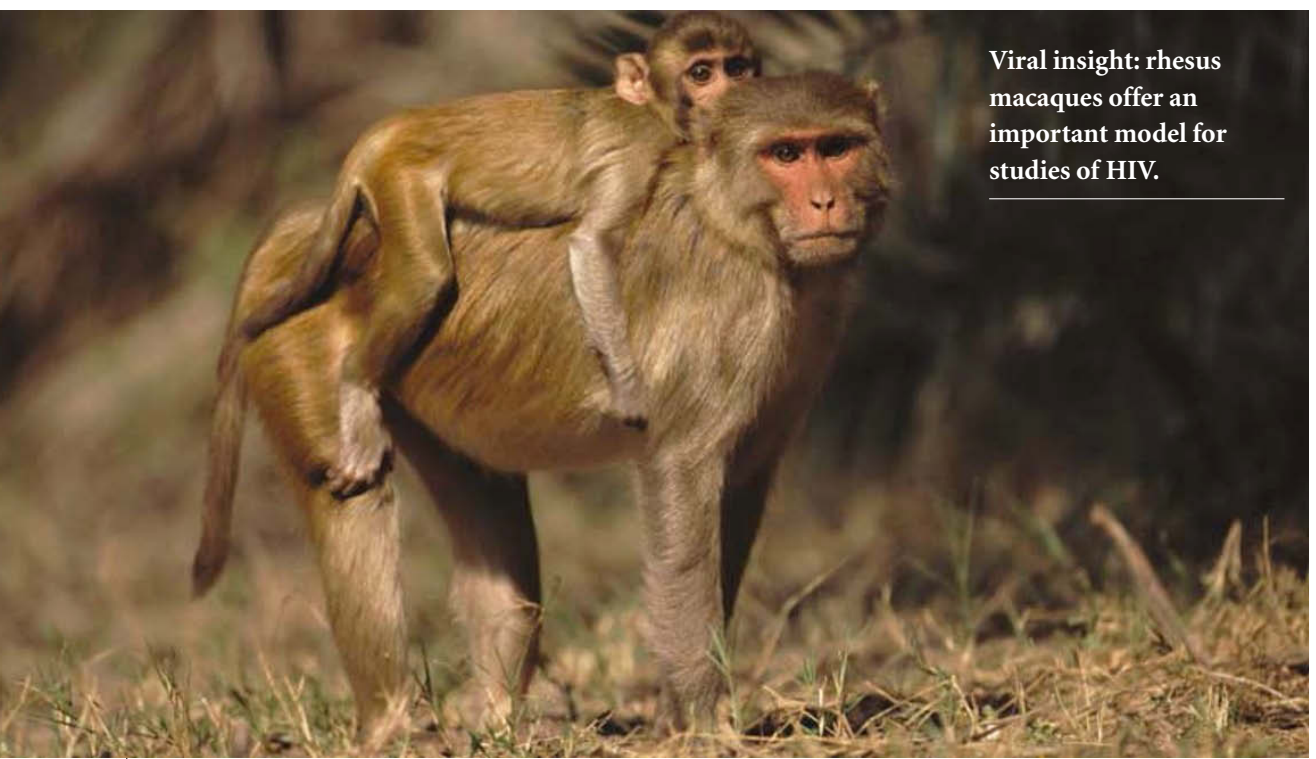

clinical trials - which means that researchers will soon be able to assess in detail the accuracy of the macaque model by comparing the results of the monkey and human trials.

Primate research is also seen as unavoidable in other areas of research with obvious medical applications. Take reproductive biology: research on new forms of oral contraception, for example, can be undertaken with rodents or sheep, because the pituitary gland at the base of the brain, responsible for the release of reproductive hormones, is broadly similar in all mammals. But attempts to understand the underlying genetic basis of human infertility rely heavily on primates.
Macaques are also vital for research into endometriosis, a painful and distressing abnormality of the menstrual cycle, because lower mammals do not menstruate.

For neuroscientists, the very attributes that make primates such an essential model — their high cognitive ability and similar brain architecture to humans - is precisely why invasive experiments are so controversial. Recent studies in macaques based on electrical recordings from individual neurons, for instance, have been key to advancing understanding of how the brain makes simple decisions based on its perception of the outside world. In combination with studies using non-invasive techniques such as functional magnetic resonance imaging, which can pinpoint regions of brain activity, electrophysiological recordings from monkeys' brains have transformed our understanding of cognitive neuroscience. Primate research is also refining neurologists' ability to interpret human brain images, and might bring important insights into a range of human diseases. "Virtually every major neuropsychiatric disorder and many neurosurgical and neurological ones involve the frontal lobes," says a British neuroscientist. "These are areas that are well developed only in primate brains."

\section{Public unease}

But despite the potential medical applications, many fundamental neuroscience studies seem arcane to non-specialists. And with images showing primates with electrodes protruding from their skulls being among the most effective recruiting tools for the animal-rights movement, neuroscientists who work on primates have an extremely difficult job in trying to win public acceptance for their work. "I suspect it is a debate that cannot be won, as it cannot be argued in rational terms only," says one British researcher.

The British public is known for its unease about animal experimentation, and the country is also home to a vicious minority of animal-rights terrorists, who have targeted scientists with letter bombs, fire bombs and envelopes booby-trapped with razor blades.

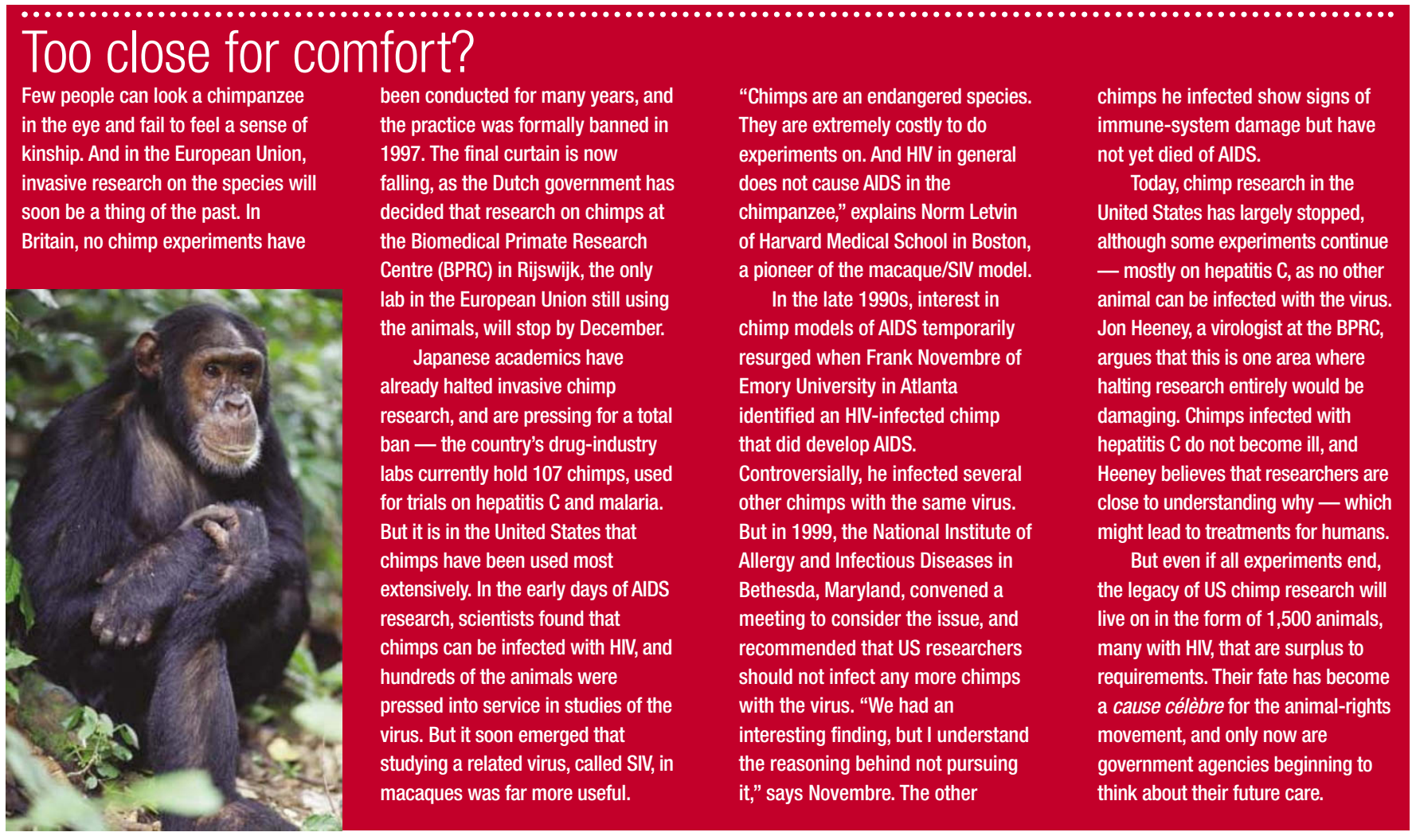


